\title{
Nonequilibrium Domain Configurations Undergoing Large Angle Rotations in Mesoscopic Magnetic Thin Film Elements
}

\author{
B. C. Choi ${ }^{1 *}$, Y. K. Hong ${ }^{2}$, J. Rudge ${ }^{1}$, G. Donohoe ${ }^{3}$, and Q. F. Xiao ${ }^{1}$ \\ ${ }^{1}$ Department of Physics \& Astronomy, University of Victoria, Victoria, BC, V8W 3P6, Canada \\ ${ }^{2}$ Department of Materials Science and Engineering, University of Idaho, Moscow, ID 83844, USA \\ ${ }^{3}$ Department of Electrical and Computer Engineering, University of Idaho, Moscow, ID 83844, USA
}

(Received 12 May 2006)

\begin{abstract}
The physical origin of complex dynamic domain configuration in nonequilibrium magnetic systems with mesoscopic length scales has been studied. An increasing complexity in the spatial feature of the evolution is found to accompany the increasing reversal speed, when a ferromagnetic element is driven by progressively faster switching fields applied antiparallel to the initial magnetization direction. As reversal rates approach the characteristic precession frequencies of spin fluctuations, the thermal energy can boost the magnetization into local configurations which are completely different from those experienced during quasistatic reversal. The sensitive dependence of the spatial pattern on switching speed can be understood in terms of a dynamic exchange interaction of thermally excited spins; the coherent modulation of the spins is strongly dependent on the rise time of switching pulses.
\end{abstract}

Key words : magnetization dynamics, nonequilibrium phenomena in magnetic domain configurations

\section{Introduction}

The physics of magnetism in small magnetic elements, where the thickness and lateral dimensions are on the nanometer scale, has become one of the most vigorous research areas in contemporary solid state physics. Recently attention has focused on the dynamic behavior of the magnetization in small patterned magnetic elements, fabricated by using electron beam lithography technique [1-10]. This is mainly because magnetization dynamics in thin continuous films on short time scales is different in many aspects from the static case [11-13]. Moreover, the magnetization dynamics in small patterned elements significantly differs from that in continuous films due to the magnetostatics of element edges, thus modifying the equilibrium states of the element in terms of the magnetic moment distribution $[14,15]$. From a practical point of view, understanding magnetization dynamics on nanoand pico-second time scales in small elements with dimensions in the micrometer size regime and below has become crucial, owing to the increasing demands on

*Corresponding author: Tel: +1-250-721-7731,

Fax:+1-250-721-7715, e-mail: bchoi@uvic.ca conventional storage technologies and for newer approaches such as magnetic random access memories (MRAM) [16, 17]. For example, the data rate for current hard disk magnetic recording device is approaching 1 Gbit/s.

For the study of the magnetization dynamics in magnetic films and small elements, it has been demonstrated that time-resolved scanning Kerr microscopy (TR-SKM) and time-resolved $\mathrm{x}$-ray magnetic circular dichroism (XMCD) are powerful tools, allowing direct insight into the spatiotemporal evolution of the switching process in small magnetic elements [3-10]. Even though the study of large-angle magnetization dynamics at the picosecond timescale in micro- and nanoscale magnets has attracted the attention of a number of research groups during the last few years, the precise physical description of the mechanism for nonequilibrium domain configurations associated with large angle rotations in the $\mathrm{GHz}$ regime still remains a challenging issue.

\section{Experiments}

In this paper, we present the results of picosecond timeresolved spin dynamics of mesoscopic $\mathrm{Ni}_{80} \mathrm{Fe}_{20}$ thin-film 
elements. A $15 \mathrm{~nm}$ thick polycrystalline $\mathrm{Ni}_{80} \mathrm{Fe}_{20}$ film was prepared by magnetron sputtering. During film deposition a magnetic field was applied, resulting in an induced uniaxial anisotropy. The elements were fabricated using electron beam lithography and lift-off, and had a fixed size of $10 \mu \mathrm{m} \times 10 \mu \mathrm{m}$. The patterned elements were made on a $20 \mu \mathrm{m}$ wide by $300 \mathrm{~nm}$ thick gold transmission line that carried a fast current pulse $[10,11]$. The current created an in-plane switching field $\left(H_{s}\right)$ of $24 \mathrm{kA} / \mathrm{m}$ in strength along the long axis of the sample. The rise time of the switching pulse was varied; $0.24 \mathrm{~ns}, 2.8 \mathrm{~ns}$, and 5.1 ns. The switching pulses were synchronously triggered by a mode-locked Ti-sapphire femtosecond laser (tuned to $\lambda=780 \mathrm{~nm}$ ) at a $0.8 \mathrm{MHz}$ repetition rate. Dynamic domain images were obtained using time-resolved scanning Kerr microscopy (TR-SKM) with a spatial resolution (Rayleigh criterion) of $800 \mathrm{~nm}$. For the measurements a so-called $180^{\circ}$ dynamic magnetization reversal configuration was used, in which the sample was first magnetically saturated by an easy-axis static bias field $\left(H_{l}=4.8\right.$ $\mathrm{kA} / \mathrm{m})$ in the plane of the sample, and a switching field pulse $\left(H_{s}=24 \mathrm{kA} / \mathrm{m}\right)$ was then applied antiparallel to $H_{l}$ in order to reverse the magnetization $[10,11]$.

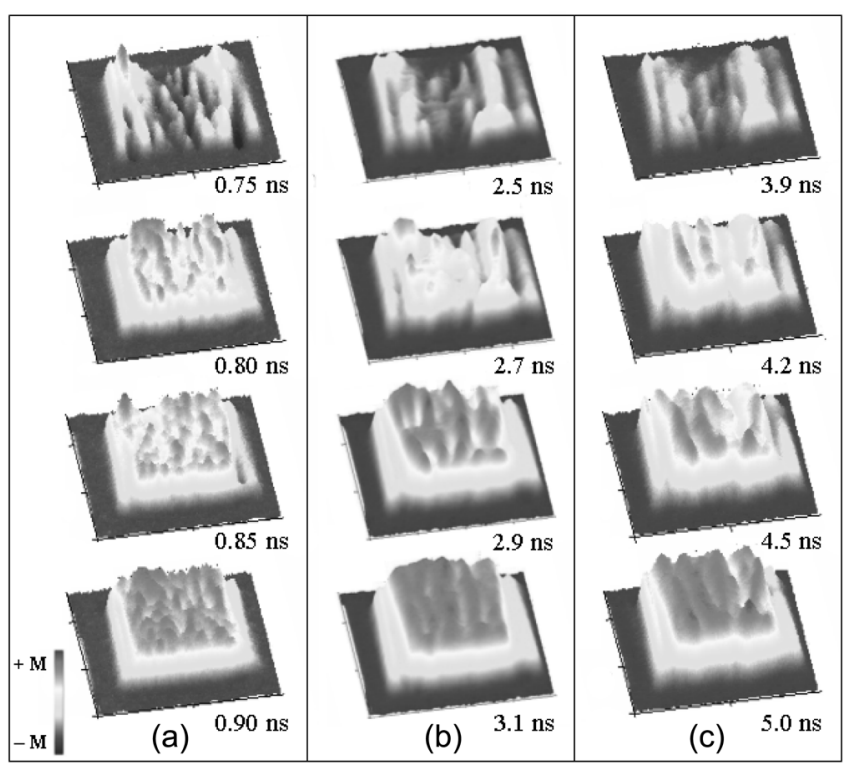

Fig. 1. Nonequilibrium domain configurations of the easy axis magnetization component $\left(M_{x}\right)$ experimentally captured at selected time points after the onset of a magnetic switching field with the rise time, $T_{\text {rise }}$, of (a) $0.24 \mathrm{ns,} \mathrm{(b)} 2.8 \mathrm{~ns}$, and (c) 5.1 ns. A complex domain pattern evolves during the reversal for $T_{\text {rise }}=0.24 \mathrm{~ns}$, unlike that observed at slower switching speeds $\left(T_{\text {rise }}=2.8 \mathrm{~ns}\right.$ and $\left.5.1 \mathrm{~ns}\right)$. The 3D topography and color map both render the magnitude of the magnetic signal from unchanged (shown as blue areas) to fully reversed (shown as red areas).

\section{Results and Discussion}

First we studied the spatiotemporal evolution of the magnetization in response to short magnetic pulses. Fig. 1 presents nonequilibrium domain structures captured at selected time points after the application of the switching

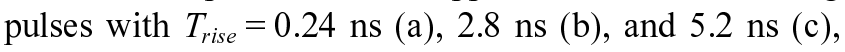
respectively. In general the dynamic behavior in the evolving magnetization configuration undergoing ultrafast $180^{\circ}$ reversal reveals a complex and non-uniform spatial appearance. For all cases studied the magnetization switching starts with the reversal of edge domains near the element borders, but the detailed temporal evolution of magnetization configurations drastically differ from each other. For the faster switching $\left(T_{\text {rise }}=0.24 \mathrm{~ns}\right)$, the emergence of the complex domain can be seen from nearly the beginning of the reversal process. At the time delay of $0.75 \mathrm{~ns}$, the magnetization reversal in progress at the ends of the platelet (along the switching field axis) is accompanied by the nucleation of branch-like fine structures in the interior regions. Complex domain structures evolve out of these nucleation sites, and once a structure forms, the completion of reversal is mainly governed by a gradual expansion of the initially reversed domains (shown for $t=0.8 \mathrm{~ns}-0.9 \mathrm{~ns}$ in Fig. 1(a)). The nonequilibrium magnetization configurations for slower switching are very different from the fast case. For $T_{\text {rise }}=2.8 \mathrm{~ns}$ and 5.1 ns, shown in Fig. 1(b) and (c), the complex domain structure is less pronounced and instead the domain configuration has a more regular character in which stripe domains predominate. For example, at the initial state for $T_{\text {rise }}=5.1 \mathrm{~ns}$, i.e., $t=3.9 \mathrm{~ns}$ in Fig. 1(c), the magnetization reversal also starts at the element ends, with stripe-like domains formed in the interior regions. With increasing time the stripe domains are fully developed and run orthogonal to the average magnetization direction (shown for $\mathrm{t}=4.2 \mathrm{~ns}-5 \mathrm{~ns}$ in Fig. 1(c)). From this comparison, we conclude that the nonequilibrium domain configurations are differently activated through the variation of the switching speed. This observation clearly illustrates that the speed at which the magnetization reversal is driven plays an essential role in determining the nonequilibrium domain patterns.

The most interesting question is how the complex spatiotemporal structure arises and how the complexity in the spatial structure of the evolution increases when a ferromagnetic element is driven by faster reversing fields. In order to shed light to the issue, micromagnetic modeling based on the Landau-Lifshitz-Gilbert (LLG) equation was carried out, with a Langevin term to represent thermal fluctuations of the magnetization for finite temperatures 


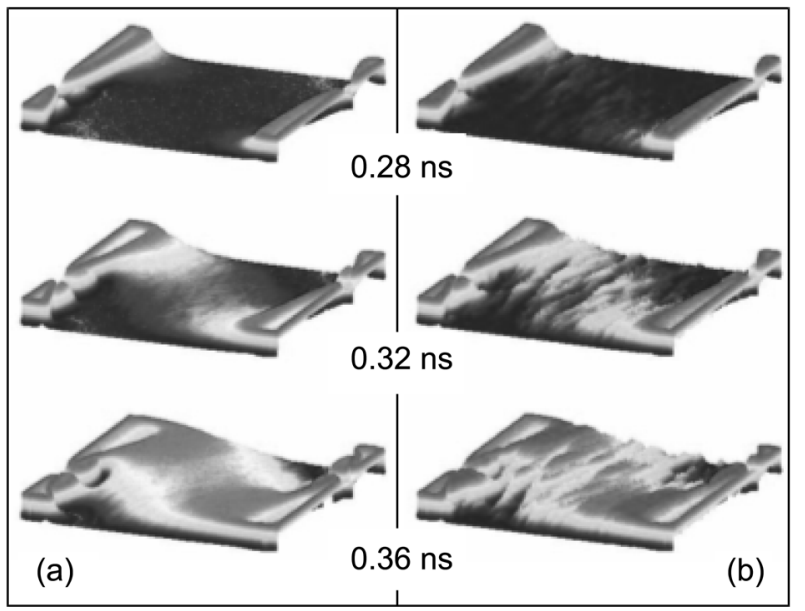

Fig. 2. Comparison of domain configurations simulated at (a) $0 \mathrm{~K}$ and (b) $300 \mathrm{~K}$. The experimentally observed non-uniform spatial feature, formed in the initial state of the magnetization reversal, becomes clearly visible when the thermal effect is included in the modeling.

[18]. The material parameters used in the modeling are magnetization saturation $M_{S}=860 \mathrm{kA} / \mathrm{m}$, exchange stiffness $A=1.33 \times 10^{-12} \mathrm{~J} / \mathrm{m}$, and the damping constant $\mathrm{a}=$ 0.01 . The unit cell volume is $9 \times 9 \times 15 \mathrm{~nm}^{3}$, which is small enough to catch the dynamic behavior of domains. The initial magnetization states are obtained by minimizing the micromagnetic energy in the presence of an external bias field of $4.8 \mathrm{kA} / \mathrm{m}$ to mimic the experimental situations, and this leads to the so called flower state of the magnetization configuration. After the initial magnetization configuration was obtained, dynamical simulations were performed by applying magnetic pulses with different rise times. Fig. 2 shows a comparison of domain configurations simulated at (a) $0 \mathrm{~K}$ and (b) $300 \mathrm{~K}$ by applying the switching pulse with $T_{\text {rise }}=0.24$ ns. The modeling at $0 \mathrm{~K}$ reproduces some characteristics of the observed domain images, in which the magnetization reversal initiated at the element edges propagates towards the sample center. The detailed spatial structures in the model, however, do not closely resemble those of the experiment. Much better agreement with experiment is found by the modeling corresponding to a $300 \mathrm{~K}$ sample temperature. In particular, the experimentally observed faint, branchlike, fine structure formed in the initial stages of the magnetization reversal becomes clearly visible in the domain images, when the simulations are carried out at $300 \mathrm{~K}$ (as shown for $t=0.28 \mathrm{~ns}$ in Fig. 2(b)). The evolution of the simulated domain configurations reveals that the complex domain structures evolve out of these fine structures, in agreement with experimental observations. From this comparison we conclude that the com-

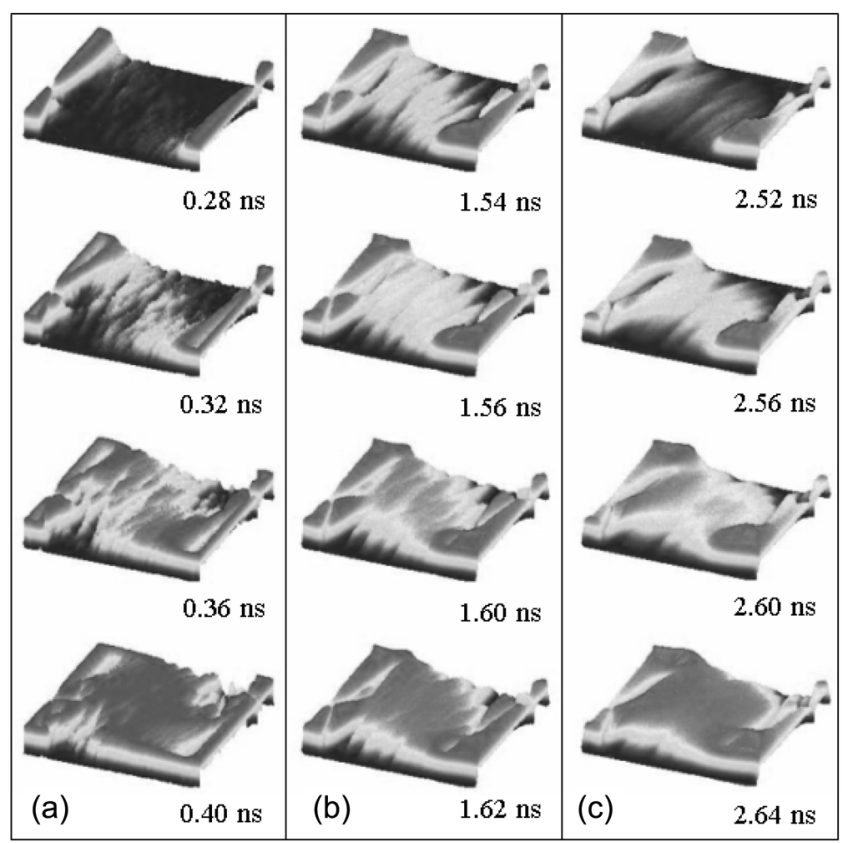

Fig. 3. Nonequilibrium domain configurations representing $M_{x}$ simulated for different magnetic pulse rise times $\left(T_{\text {rise }}=0.24\right.$ ns (a), $2.8 \mathrm{~ns}$ (b), and $5.1 \mathrm{~ns}$ (c)). The experimentally observed sensitive dependence of the spatial pattern on switching speed is well reproduced in the modeling.

plex domain patterns are activated through the thermal fluctuations. We note that we can stochastically treat thermal fluctuations, since the characteristic time of thermally excited spin fluctuations is of the order of $10^{-13}$ $\mathrm{s}$ at room temperature and is much smaller than the response time of the magnetization, which is on the order of $10^{-9} \mathrm{~s}$ [19]. Thermally activated spin fluctuations quickly modulate each other via the exchange interaction and form local in-phase regions, which are manifested as the faint branchlike fine structure formed in the initial state of the magnetization reversal. This mechanism can be extended to the description for the systematic evolution of the domain patterns to progressively faster switching fields. In the case of relatively slow switching, e.g., $T_{\text {rise }}=2.8 \mathrm{~ns}$ and $5.1 \mathrm{~ns}$ (Fig. 3(b) and (c)), the thermally excited spins modulate long enough to create long stripe patterns, while complete stripe patterns cannot be formed for $T_{\text {rise }}=0.24 \mathrm{~ns}$ due to the limited time for completing modulation across the sample (Fig. 3(a)). Instead, labyrinthlike patterns, which are irregular-shaped regions due to uncompleted modulation processes, appear. Since such modulation is mediated by the exchange interaction of precessing spins, the characteristic time of modulation is given by the ferromagnetic resonance frequency, which is in the range of a few $\mathrm{GHz}$ in typical ferromagnetic materials. The characteristic spin modulation time can, 

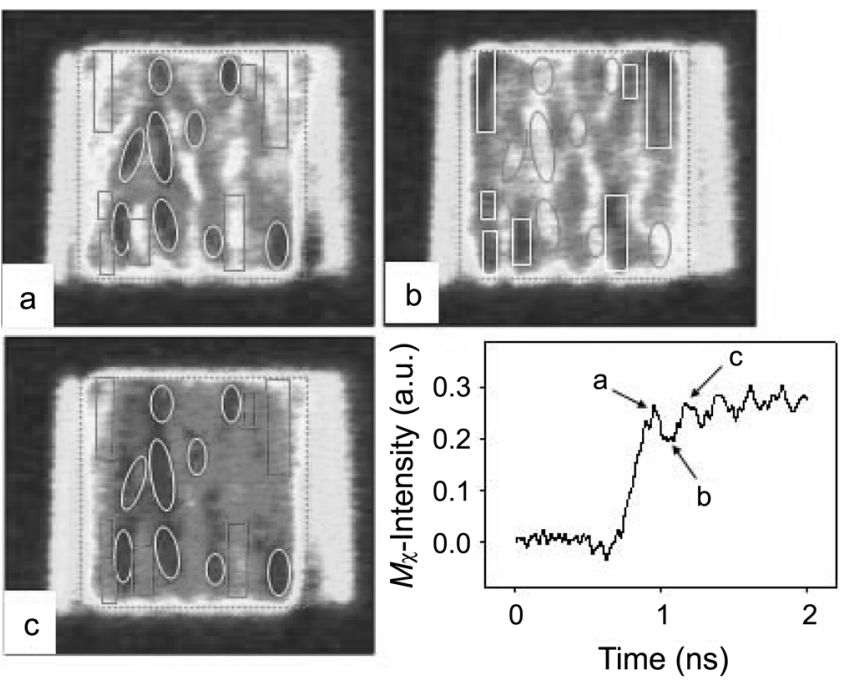

Fig. 4. Comparing spatiotemporally resolved domain images measured at the peaks (a) and (c) and dip (b) during magnetization oscillation. Through a cycle of oscillations, the already fully reversed regions at the oscillation peak (a) simply turn into not-fully reversed regions at the dip (b). In the next oscillation peak (c), one observes the same pattern with progressing reversal. (Inset): Temporal evolution of the inplane magnetization component measured at the center of the sample.

therefore, be estimated to lie in the range below one nanosecond. The results from numerical modeling in Fig. 2 are consistent with our experimental observations (Fig. 1), where the characteristic stripe length scale reduces with increasing switching speed. This indicates that our modeling successfully captures the relevant physics underlying the formation of complex nonequilibrium domains. We note that the magnetization reversal process in simulation is faster than experimental cases due to the assumption of an element without any imperfections.

The precessional behavior of the dynamic domain pattern is illustrated in Fig. 4, where a series of domain images are captured during the reversal. The bottom right panel shows the temporal evolution of the magnetization component $\left(M_{x}\right)$ along the $x$ axis, measured in the center of the elements. The time trace shows an abrupt change of magnetization from one saturated state to another, and one observes the periodic change of $M_{x}$ that results from magnetization precession about the effective switching field axis. In this situation, the oscillations rapidly decay with increasing time, indicating a relatively strong effective damping. In the domain images captured during the oscillation, some of the fine spatial structure has vanished before even the first cycle of precession is complete, but an alternating change of domain pattern contrast is clearly observed during the subsequent evolution, without signifi-

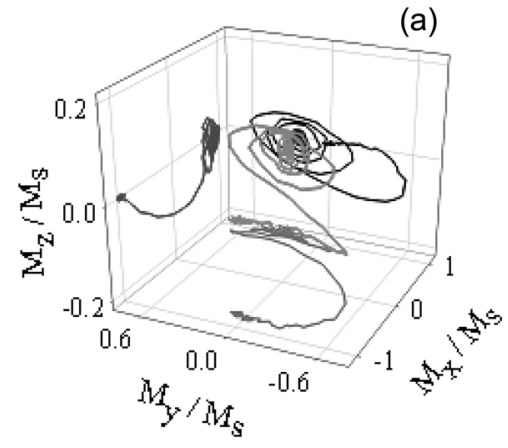

(b)

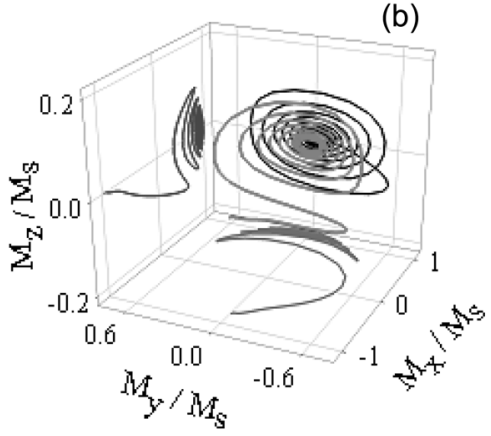

Fig. 5. Comparison of the actual directions of the magnetization vector during a reversal process. Measured (a) and simulated (b) views of 3D magnetization trajectories and corresponding projections are shown. In both cases the magnetic element is excited by the application of the switching pulse with $T_{\text {rise }}=2.8 \mathrm{~ns}$.

cant changes in the pattern shape. Selecting frames according to the time traces in the inset (peak (a), dip (b), peak (c)) one sees an oscillation of the entire pattern superimposed upon the completion of the magnetization reversal. During oscillations, the already fully reversed regions, measured at the oscillation peak (a), simply turn into not-fully-reversed regions at the dip (b). In the next oscillation peak (c), one observes the same domain pattern as the reversal progresses. This observation implies that the switching mechanism is driven by the multiple local nucleation of reversed domains, and the domain walls seem not to be able to propagate far enough across the sample in order to build bigger reversed domains. Instead, collective precessional motion occurs inside the reversed domains. This implies that the selected domain pattern is maintained while the remaining excess Zeeman energy introduced by the applied switching field is dissipated by carrying out the precessional magnetization motion.

The actual directions of the magnetization vector during precession are directly compared in Fig. 5, where the measured (a) and simulated (b) and measured (c) views of 3D magnetization trajectories and corresponding projections are shown. In both cases the magnetic element is 
excited by the application of the switching pulse with $T_{\text {rise }}$ $=2.8 \mathrm{~ns}$. Both trajectories reveal an initial rapid rotation along regular trajectories, and the torque induced by the switching pulse tilts the magnetization vector out of the $x$ $y$ plane. After the main reversal process, i.e., $M_{x} / M_{s}$ from -1 to +1 , the magnetization trajectories follow a rotational path mainly around the $z$ axis implying the precessional motion of the magnetization. The magnetization precession persists until the remaining excess Zeeman energy introduced by the applied switching pulse vanishes. Slightly different from experiments, the precessional motion of the magnetization in the model is more pronounced due to the assumption of an element without any imperfections.

\section{Conclusion}

In conclusion, we report on the spatiotemporal nature of the magnetization reversal of a small $\mathrm{Ni}_{80} \mathrm{Fe}_{20}$ element to progressively faster switching fields. In general, the magnetization evolves into a complex domain structure in nonequilibrium state when a magnetic element is excited by applying a short magnetic field pulse. The degree of complexity of the nonequilibrium domain structure is found to be a strong function of the speed of the switching process. The sensitive dependence of the spatial features on switching speed is attributed to the dynamic exchange interaction of thermally excited spins, in which the coherent modulation of the spins is strongly dependent on the rise time of switching pulses.

\section{Acknowledgements}

We gratefully acknowledge support from the Canada
Foundation for Innovation (CIF), the British Columbia Knowledge Development Fund (BCKDF), the Natural Sciences and Engineering Research Council (NSERC) of Canada, and the U.S. Air Force Research Laboratory (AFRL) under Grant F29601-04-1-206.

\section{References}

[1] K. Y. Guslienko et al., Phys. Rev. Lett. 96, 67205 (2006).

[2] T. Devolder et al., Appl. Phys. Lett. 86, 62505 (2005).

[3] D. Chumakov et al., Phys. Rev. B 71, 14410 (2005).

[4] A. Neudert, J. MvVord, R. Schaefer, and L. Schultz, J. Appl. Phys. 97, 10E701 (2005).

[5] J. Vogel et al., Phys. Rev. B 72, 220402(R) (2005).

[6] V. V. Kruglyak et al., Phys. Rev. B 71, 220409(R) (2005).

[7] B. C. Choi, J. Ho, G. Arnup, and M. R. Freeman, Phys. Rev. Lett. 95, 237211 (2005).

[8] S. Choe, Y. Acremann, A. Scholl, A. Bauer, A. Doran, J. Stohr, and H. A. Padmore, Science 304, 420 (2004).

[9] C. M. Schneider et al., Appl. Phys. Lett. 85, 2562 (2004).

[10] B. C. Choi, G. E. Ballentine, M. Belov, W. K. Hiebert, and M. R. Freeman, Phys. Rev. Lett. 86, 728 (2001).

[11] B. Heinrich and J. A. C. Bland (Eds.), "Ultrathin Magnetic Structures", Springer Verlag, (1994-2005).

[12] S. W. Yuan and H. N. Bertram, J. Appl. Phys. 73, 5992 (1993).

[13] M. N. Baibich et al., Phys. Rev. Lett. 61, 2472 (1988).

[14] A. F. Popkov, L. L. Savchenko, N. V. Vorotnikova, S. Tehrani, and J. Shi, Appl. Phys. Lett. 77, 277 (2000).

[15] K. J. Kirk, J. N. Chapman, and C. D. W. Wilkinson, Appl. Phys. Lett. 71, 539 (1997).

[16] J. M. Daughton et al., Thin Solid Films 216, 162 (1992).

[17] W. J. Gallagher et al., J. Appl. Phys. 81, 3741 (1997).

[18] W. F. Brown, Micromagnetics. New York, Wiley (1963).

[19] W. F. Brown, Phys. Rev. 130, 1677 (1963). 